Published in final edited form as:

Nat Rev Gastroenterol Hepatol. ; 8(9): 512-522. doi:10.1038/nrgastro.2011.131.

\title{
Clinical diagnosis and staging of cholangiocarcinoma
}

\author{
Boris Blechacz, Mina Komuta, Tania Roskams, and Gregory J. Gores \\ Division of Gastroenterology and Hepatology, College of Medicine, Mayo Clinic, 200 First Street, \\ Rochester, MN 55905, USA (B. Blechacz, G. J. Gores). Morphology and Molecular Pathology \\ Section, Katholieke University Leuven, University Hospital Street Rafael, Minderbroederstraat 12, \\ B-3000 Leuven, Belgium (M. Komuta, T. Roskams)
}

\begin{abstract}
Cholangiocarcinoma is the most frequent biliary malignancy. It is difficult to diagnose owing to its anatomic location, growth patterns and lack of definite diagnostic criteria. Currently, cholangiocarcinoma is classified into the following types according to its anatomic location along the biliary tree: intrahepatic, perihilar or distal extrahepatic cholangiocarcinoma. These cholangiocarcinoma types differ in their biological behavior and management. The appropriate stratification of patients with regard to the anatomic location and stage of cholangiocarcinoma is a key determinate in their management. Staging systems can guide this stratification and provide prognostic information. In addition, staging systems are essential in order to compare and contrast the outcomes of different therapeutic approaches. A number of staging systems exist for cholangiocarcinoma - several early ones have been updated, and new ones are being developed. We discuss the emerging diagnostic criteria as well as the different staging systems for cholangiocarcinoma, and provide a critical appraisal regarding these advances in biliary tract malignancies.
\end{abstract}

\section{Introduction}

Cholangiocarcinoma is the most common primary malignancy of the biliary tract. ${ }^{1}$ This cancer has been classified as either intrahepatic or extrahepatic, with the second-order bile ducts acting as the separation point. ${ }^{2}$ Classically, extrahepatic cholangiocarcinoma has been divided into perihilar and distal extrahepatic cholangiocarcinoma at the level of the cystic duct. This anatomical landmark to distinguish these two types of extrahepatic cholangiocarcinoma was somewhat randomly chosen, and the usefulness of this could be debated given the interindividual variation in the insertion point of the cystic into the common bile duct. However, in our opinion, perihilar and distal extrahepatic cholangiocarcinoma should be viewed as independent entities because of their distinct biology and management (Figure 1). In this classification, we include the second-order branches of the bile ducts within the perihilar diagnostic classification.

Perihilar cholangiocarcinoma is the most common type of cholangiocarcinoma, followed by the distal extra-hepatic and then the intrahepatic forms. ${ }^{3}$ The incidence of intrahepatic cholangiocarcinoma has increased over the past three decades while the incidence of

\footnotetext{
(C) 2011 Macmillan Publishers Limited. All rights reserved Correspondence to: G. J. Gores gores.gregory@mayo.edu.

Author contributions: B. Blechacz and G. J. Gores contributed to all aspects of this Review. M. Komuta contributed to the writing and editing; T. Roskams contributed to the discussion of content, writing and editing of this Review.

Supplementary information: Supplementary information is linked to the online version of the paper at www.nature.com/nrgastro

Competing interests: The authors declare no competing interests.
} 
perihilar and distal extrahepatic cholangiocarcinoma has remained stable; ${ }^{1,4}$ the reasons for this are not understood. The prognosis of this malignancy is dismal owing to its silent clinical character, difficulties in early diagnosis and limited therapeutic approaches; median survival is less than 24 months. Surgical management is the only potentially curative treatment, but is limited to early-stage disease. Several staging systems exist but the majority of these have proven insufficient for stratifying patients in alignment with therapeutic options. They also lack prognostic accuracy and/or external validation. Hence, there is an urgent need for new staging systems that will enable optimal therapeutic allocation and prognostication. We review the diagnostic criteria for cholangiocarcinoma and discuss different staging systems, including old ones as well as newly developed systems.

\section{Diagnostic criteria}

Cholangiocarcinoma is difficult to diagnose owing to its silent clinical character, the low specificity of most diagnostic modalities and the lack of absolute diagnostic criteria. The majority of patients with cholangiocarcinoma develop symptoms only at an advanced stage of disease, and the clinical presentation depends upon tumor stage, tumor location and growth pattern. Diagnosis requires a high level of suspicion in the appropriate clinical setting and a confirmatory constellation of clinical, laboratory, endoscopic and radiologic data. The majority of patients with cholangiocarcinoma develop this malignancy de novo. However, certain conditions and risk factors have been associated with an elevated risk of developing the disease (Box 1). Primary sclerosing cholangitis (PSC) is the most wellknown of these conditions and is associated with a prevalence of cholangiocarcinoma of 5$15 \% .{ }^{5}$ In patients with any of these conditions, symptoms and clinical signs associated with cholangiocarcinoma should prompt an aggressive diagnostic work-up as outlined below.

\section{Intrahepatic cholangiocarcinoma}

The Liver Cancer Study Group of Japan (LCSGJ) used retrospective data from 245 patients to distinguish three macroscopic growth types for intrahepatic cholangiocarcinoma: massforming type; periductal-infiltrating type; and intraductal-growth type (Figure 2) ${ }^{6}$ The mass-forming type is defined as a definite mass located in the liver parenchyma and is the most common form of intrahepatic cholangiocarinoma. ${ }^{7}$ The mass-forming type tends to invade the hepatic parenchyma via the portal venous system and invades lymphatic vessels at advanced stages. ${ }^{8}$ The periductal-infiltrating type is described as extending mainly longitudinally along and within the bile duct, often resulting in dilatation of the peripheral bile ducts. The periductal-infiltrating type tends to spread along the Glisson sheath via the lymphatic vessels. The intraductal-growth type proliferates towards the lumen of the bile duct like an intraductal tumor thrombus; this form of cholangiocarcinoma often has papillary growth characteristics. Tumors not following any of these criteria are grouped into a fourth category as 'undefined'. Tumors with more than one of the above described growth types are described by the corresponding descriptions connected by ' + ' (for example, massforming + periductal-infiltrating). ${ }^{6}$ Evidence indicates that the mass-forming + periductalinfiltrating type has a worse prognosis than other types, with higher rates of recurrence following resection. ${ }^{9,10}$ The American Joint Cancer Committee/Union for International Cancer Control (AJCC/UICC) classifies cholangiocarcinoma into the mass-forming, periductal-infiltrating and a mixed type but not the intraductal-growth type. ${ }^{11}$

Clinically, intrahepatic cholangiocarcinoma presents with unspecific symptoms such as abdominal pain, malaise, night sweats and cachexia. ${ }^{12}$ Makuuchi and colleagues have suggested that primary hepatic malignancies can be identified correctly on the basis of imaging data. ${ }^{13}$ In our experience, intrahepatic cholangiocarcinoma is the most common etiology of primary, malignant intra-hepatic mass lesions in the absence of other primary solid malignancies or cirrhosis. Several imaging modalities are being used in the evaluation 
of primary hepatic masses. ${ }^{14} \mathrm{CT}$ and MRI are both helpful for the detection of the primary tumor but both imaging techniques have low specificity. ${ }^{15}$ The sensitivity and image quality of MRI can be increased by the use of ferumoxide enhancement, although this agent is no longer available commercially. ${ }^{16,17}$ In particular, dynamic CT or MRI can help to distinguish intrahepatic cholangiocarcinoma from hepatocellular carcinoma (HCC). Up to $81 \%$ of intrahepatic cholangiocarcinomas are characterized by a progressive contrast uptake throughout the arterial and venous phase, and especially in the delayed venous phase images; this feature is in contrast to HCC, which is characterized by rapid contrast enhancement during the arterial phase and prompt washout in the delayed venous phase. ${ }^{18}$ MRI and CT are comparable in the detection of satellite lesions but CT is preferable for the depiction of vascular encasement. ${ }^{19}$ The sensitivity of PET for the detection of massforming intra-hepatic cholangiocarcinoma of $>1 \mathrm{~cm}$ diameter has been reported as 85-95\%, with a sensitivity of $100 \%$; its sensitivity and specificity for detection of nodal and distant metastatic disease is $100 \%$ and $94 \%$, respectively. ${ }^{20}$

In problematic cases, determination of the serum CA 19-9 concentration can be helpful. However, the sensitivity and specificity of this biomarker for intrahepatic cholangiocarcinoma is only $62 \%$ and $63 \%$, respectively, and should, therefore, only be used for further confirmation. Other serum markers, such as serum cytokeratin-19 fragment (CYFRA 21-1) and CA242, have been reported to have higher specificities than CA 19-9 for intrahepatic cholangiocarcinoma in a limited number of studies, but are not in routine use. $^{21-23}$

It should be emphasized that imaging is not always reliable for the diagnosis of some intrahepatic cholangiocarcinomas. For instance, intrahepatic cholangiocarcinomas $<2 \mathrm{~cm}$ in size mimic HCC because of the absence of the progressive enhancement pattern. ${ }^{18}$ The need for a liver biopsy and a histopathologic diagnosis depends upon the clinical setting and preoperative imaging. In patients with cirrhosis and an indeterminate lesion within Milan criteria, a biopsy is justified because the distinction between HCC and cholangiocarcinoma changes management (patients with intrahepatic cholangiocarcinoma should not undergo liver transplantation) and could thus have an important effect on outcome. In noncirrhotic patients who qualify for surgery, a liver biopsy may not be required if a decision has been made to proceed with surgical resection. However, in patients who are not candidates for surgical treatment and, therefore, curative treatment, a liver biopsy is usually performed before initiation of systemic therapy.

According to the AJCC/UICC ${ }^{11}$ and WHO classification systems, ${ }^{24}$ intrahepatic cholangiocarcinoma can arise from biliary epithelia at any portion of the intrahepatic biliary system, from the segmental bile ducts (lined by mucin-producing cylindrical cells) to the ductules (lined by cuboidal cholangiocytes without mucin production). ${ }^{25,26}$ Mucinproducing cells can give rise to mucin-producing tubular adenocarcinoma with or without micropapillary structures (Figure 3a). ${ }^{24}$ These features are also observed in perihilar cholangiocarcinoma and distal extrahepatic cholangiocarcinoma, as their tumor locations (liver hilar bile duct and extrahepatic bile duct) are lined with a similar phenotype of cholangiocytes. On the other hand, ductular-related intrahepatic cholangiocarcinoma can present with mixed hepatocellular and/or cholangiocellular features, since ductules are composed of hepatic progenitor cells capable of differentiating to both hepatocytes and cholangiocytes. ${ }^{27}$ For example, cholangiolocellular carcinoma, which is thought to originate from ductules, presents with mixed histopathological features, including both an HCC-like area and a mucin-producing cholangiocarcinoma area (Figures $3 \mathrm{~b}$ and $3 \mathrm{c}$ ). ${ }^{27}$ Imaging these cancers shows mixed histopathological features indicating both HCC and cholangiocarcinoma patterns. ${ }^{28,29}$ In addition, cholangiocellular carcinoma can occur in chronic liver disease conditions, and mimicking the HCC image pattern can lead to 
problems for preoperative diagnosis. Thus, there is a need to address the issue of preoperative diagnosis of intrahepatic cholangiocarcinoma.

Other rare histologic types have been included in the $7^{\text {th }}$ edition of the AJCC/UICC staging system (Box 2). ${ }^{11}$ However, it is questionable if these should be included in the same category as cholangiocarcinoma as these malignancies originate from different cell types and can, therefore, be expected to display different biologic behavior. Hence, prognostic information observed in adenocarcinomas might not be reliable in these cancers. The accuracy of distinguishing metastatic adenocarcinomas can be improved by immunoprofiling with a combination of cytokeratin (K) 7 and K20 immuno-histochemical staining. ${ }^{30,31}$ However, metastatic liver tumors, especially from pancreatic cancers, are still challenging to diagnose. The use of liver biopsy is limited by its potential complications, including hemorrhage and tumor spread, as well as by its frequently unspecific histopathology. Makuuchi and colleagues have suggested that the diagnosis of an intrahepatic cholangiocarcinoma can be made with up to $100 \%$ accuracy based upon a combination of clinical presentation, laboratory analysis and radiologic evaluation. ${ }^{13}$ However, the Barcelona liver cancer group have presented the diagnostic problem that intrahepatic cholangiocarcinoma in patients with cirrhosis is difficult to diagnose radiographically, ${ }^{18,32}$ requiring histopathology for definitive diagnosis.

In summary, clinically, intrahepatic cholangiocarcinoma can be assumed on the basis of its venous phase contrast enhancement on dynamic imaging in the absence of other, extrahepatic primary malignancies and cirrhosis. Tumor markers such as CA 19-9 can be used for additional evidence but are not sufficient for diagnosis. However, for a definitive diagnosis of intrahepatic cholangiocarcinoma, a histological diagnosis is required.

Intrahepatic cholangiocarcinoma arising from ductules may present with mixed histological and imaging features of both HCC and cholangiocarcinoma. Patients with intrahepatic cholangiocarcinoma or mixed HCC/intrahepatic cholangiocarcinoma should not undergo liver transplantation because of the risk of suboptimal outcomes. ${ }^{33}$ A diagnostic approach for intrahepatic cholangiocarcinoma is depicted in Figure 4.

\section{Perihilar cholangiocarcinoma}

Although the definition of intrahepatic cholangiocarcinoma is the same in both the UICC ${ }^{11}$ and WHO classification, ${ }^{24}$ the definition of perihilar cholangiocarcinoma differs between the two. According to the UICC classification, ${ }^{11}$ perihilar cholangiocarcinoma is proximally separated from intrahepatic cholangiocarcinoma by the second-order bile ducts, and is distally separated from distal extrahepatic cholangiocarcinoma by the insertion of the cystic duct into the extrahepatic biliary tree. By contrast, the WHO classification defines hilar cholangiocarcinoma, often called 'Klatskin tumor', as arising from the right and left hepatic ducts at or near their junction, but does not include the level of cystic bile duct insertion. ${ }^{24}$ On the other hand, perihilar intrahepatic cholangiocarcinoma defined by the WHO classification corresponds to intrahepatic cholangiocarcinoma arising from the intrahepatic large bile ducts. These definitions are confusing. Given the differences in biology and management, we believe perihilar cholangiocarcinoma is a separate entity from intrahepatic cholangiocarcinoma and distal extrahepatic cholangiocarcinoma.

Macroscopically, perihilar cholangiocarcinoma can display an exophytic or an intraductal growth pattern, with the former either displaying a nodular or a periductal-infiltrating anatomy. ${ }^{34}$ The periductal-infiltrating form of this cancer is the most common; as the tumor progresses it looses its tropism for bile and then forms a mass-forming + periductalinfiltrating lesion. Histopathologically, the majority of perihilar cholangiocarcinomas are adenocarcinomas that are moderately to well differentiated with mucin production and abundant fibrous stroma. ${ }^{35-37}$ Perihilar cholangiocarcinoma often spreads by perineural 
invasion and lymph node involvement (Figure 3d). As perihilar cholangiocarcinoma involves the main bile duct, intrahepatic cholangitis and/or secondary sclerosing-cholangitislike features are seen in the nontumoral liver tissue. In addition, $90 \%$ of patients with perihilar cholangiocarcinoma clinically present with biliary problems such as painless jaundice, and $10 \%$ of patients present with cholangitis. ${ }^{38,39}$ Therefore, perihilar cholangiocarcinoma can be diagnosed earlier and at a smaller size than intrahepatic cholangiocarcinoma. Systemic signs of malignancy (that is, weight loss, anorexia and fatigue) are observed in up to $56 \%$ of patients with perihilar cholangiocarcinoma at presentation. ${ }^{39}$ Unilateral hepatic lobe hypertrophy with contralateral hepatic lobar atrophy, which is known as a hypertrophy-atrophy complex, is secondary to unilobar biliary obstruction often with ipsilateral vascular encasement. On physical examination, this may present as a palpable prominence of one hepatic lobe. ${ }^{40}$ Patients presenting with the above signs and symptoms - in particular, in patients with known risk factors as outlined in Box 1 - should be evaluated for cholangiocarcinoma. Figure 5 presents an algorithm for the management of patients who present with a malignant hilar biliary obstruction. Laboratory analysis is mostly unspecific and reflects the associated cholestasis and cholangitis. ${ }^{41}$ Immunoglobulin G4 (IgG4) cholangiopathy should be ruled out by evaluation for increased IgG4 in serum and biliary samples. ${ }^{42}$ Tumor markers, in particular serum CA 19-9 determination, can be helpful in cases of indeterminate biliary strictures but are not diagnostic. Serum CA 19-9 levels can be increased in patients with cholangitis, which can be surprisingly indolent in patients who have biliary stents in place. ${ }^{43}$ In patients with PSC, the sensitivity and specificity of CA $19-9$ is $79 \%$ and $98 \%$, respectively, at a serum concentration of $>129 \mathrm{U} / \mathrm{ml}^{44}$ In patients who do not have PSC, a CA 19-9 serum concentration of $>100 \mathrm{U} / \mathrm{ml}$ has a sensitivity of $76 \%$ and a negative predictive value of $92 \%$ compared with patients who have benign strictures. ${ }^{45}$ Of note, $10 \%$ of individuals lack the Lewis antigen and do not produce CA 19-9, and occasionally tumor cells lose the ability to express a tumor marker. ${ }^{46}$

Radiologic evaluation is critical for detection and evaluation of tumor extent, as well as for preoperative planning. In the past, the use of CT was limited due to its low accuracy. ${ }^{47}$ However, technical advances, such as multidetector technology and multiphase scanning, have significantly improved the accuracy of CT in perihilar cholangiocarcinoma. ${ }^{19}$ The accuracy for detection of portal vein involvement has been reported to be up to $87 \%$ and for arterial involvement as high as $93 \% .{ }^{48}$ The accuracy of CT in the assessment of resectability has been reported as $60-88 \%$ with negative predictive values of $85-100 \% .{ }^{49,50}$ However, its sensitivity for detection of regional lymph node metastases is only $54 \%$ and CT tends to underestimate the extent of the proximal tumor. ${ }^{19,48,51}$ Magnetic resonance cholangiopancreatography (MRCP) is the imaging modality of choice for perihilar cholangiocarcinoma. Its accuracy in assessing local extent and resectability is up to $95 \%$, and is comparable to endoscopic retrograde cholangiopancreaticography (ERCP). ${ }^{52-55}$ However, its accuracy in the assessment of vascular involvement and hepatic parenchyma invasion is only $67-73 \%$ and $75-80 \%$, respectively. ${ }^{53,54}$ The sensitivity and specificity of PET for perihilar cholangiocarcinoma is only $69 \%$ and $67 \%$, respectively; for the detection of regional lymph node metastases, its sensitivity is $13-38 \% .51,56$ The role of PET is further limited due to false-positive results in the setting of inflammation and false-negative results due to the high desmoplastic reaction that occurs in these tumors. ${ }^{14}$ Invasive diagnostic tests, such as ERCP, percutaneous transhepatic cholangiography (PTC) and endoscopic ultrasonography (EUS), have an important diagnostic and therapeutic role in perihilar cholangiocarcinoma. ERCP and PTC allow assessment of strictures, sampling of biliary epithelial cells and therapeutic dilatation and stent placement. EUS has the highest sensitivity for the assessment of regional lymphadenopathy and allows fine needle aspiration of suspicious lymph nodes; however, the primary tumor should not be biopsied as discussed below. ${ }^{38}$ 
A tissue diagnosis of perihilar cholangiocarcinoma is difficult for several reasons. First, techniques for sampling the biliary tree, usually endoscopically directed brushings, frequently provide only a limited number of cells such that sampling errors can result in false-negative results. Also, desmoplasia limits the number of cells obtained by brush cytology. Hence, the sensitivity of conventional cytology in perihilar cholangiocarcinoma is approximately $20 \% .{ }^{57}$ The vast majority of perihilar cholangiocarcinomas are periductal, infiltrating cancers, and as such do not demonstrate mass lesions on cross-sectional imaging studies. The lack of a mass lesion precludes percutaneous biopsy techniques. Even in the presence of a hilar mass, we discourage percutaneous or EUS-guided biopsies of primary lesions in patients who are potential candidates for treatment with curative intent because of the potential for tumor spread. For example, the Mayo Clinic transplant protocol for perihilar cholangiocarcinoma excludes patients who have undergone biopsy of the primary tumor. Given the limitations of conventional cytology, additional cytologic approaches are needed to diagnose this cancer. Chromosomal analysis using fluorescent in situ hybridization (FISH) has been established as an additional test for biliary tissue samples, with a resulting sensitivity of $47 \%$ and a specificity of $97 \%$ for detection of cholangiocarcinoma in patients with PSC. ${ }^{58}$ In FISH analysis, three subsets of chromosomal amplification can occur: trisomy 7; tetra-somy or duplication of all chromosomes labeled; and polysomy or amplification of at least two chromosomes beyond tetrasomy. Polysomy is observed in up to $77 \%$ of cholangiocarcinoma cases, ${ }^{59}$ and we consider the combination of a dominant stricture and polysomy as a diagnostic criteria for this disease. Trisomy 7 describes an amplification of chromosome 7 alone; it is frequently observed in inflammatory conditions and, although not diagnostic for cholangiocarcinoma, it is associated with an increased risk of its development over time and warrants close follow-up. Tetrasomy must be interpreted with caution, because high mitotic rates yield tetrasomy during the $\mathrm{M}$ phase of the cell cycle.

In summary, the diagnostic criteria for perihilar cholangiocarcinoma consist of a constellation of different clinical data. These include a dominant stricture in the perihilar biliary tree with either the presence of adenocarcinoma cells revealed by conventional cytology and/or polysomy on FISH analysis of cytologic specimens. In the absence of cytologic abnormalities - a frequent problem in the clinical setting owing to the low sensitivity of cytologic techniques and sampling errors-a hilar mass on axial imaging with an associated biliary stricture, and/or hypertrophy-atrophy complex can also serve as a combination diagnostic for perihilar cholangiocarcinoma. Vascular encasement is almost always a malignant feature. Serum levels of CA 19-9 can help to provide further evidence but are not sufficient as a sole diagnostic criterion; very high serum levels of this marker in the absence of cholangitis can be indicative of metastatic disease (for example, peritoneal carcinomatosis).

\section{Distal extrahepatic cholangiocarcinoma}

Distal extrahepatic cholangiocarcinomas are defined as growing along the common bile duct between the cystic duct and the ampulla of Vater; however, they are clearly separated from ampullary carcinomas. Histologically, they are predominantly well-to-moderately differentiated adenocarcinomas. ${ }^{24}$ The latest WHO classification proposes two types of precursor lesions; intraductal papillary neoplasms and biliary intraepithelial neoplasia (BillN). ${ }^{24}$ Clinically, distal cholangiocarcinomas present similarly to perihilar cholangiocarcinoma - causing symptoms of cholestasis and cholangitis. ${ }^{36,60}$ Although jaundice, abdominal pain and weight loss are observed at equal rates for perihilar and distal extrahepatic cholangiocarcinoma, fever is more common in perihilar cholangiocarcinoma. ${ }^{41}$ Lymph node metastases are less common than in perihilar cholangiocarcinoma. ${ }^{28}$ In most clinical studies evaluating diagnostic modalities, perihilar and distal extrahepatic 
cholangiocarcinoma have been grouped together as extrahepatic cholangiocarcinoma. Therefore, there are no studies evaluating the different tumor markers and radiologic modalities for distal extrahepatic cholangiocarcinoma as a separate entity. Given the similar clinical presentation, we apply the same diagnostic criteria described above for perihilar cholangiocarcinoma. However, we stress that perihilar cholangiocarcinoma and distal extrahepatic cholangiocarcinoma are separate entities in their biology and treatment, and should, therefore, be treated as such.

In summary, the diagnosis of distal extrahepatic cholangiocarcinoma requires the presence of a dominant stricture and a positive cytology for adenocarcinoma cells and/or polysomy on FISH. Mass lesions are unusual in this type of cholangiocarcinoma.

\section{Staging of cholangiocarcinoma}

The purpose of staging systems for malignancies is to provide information on the prognosis and natural history of a malignancy, guidance for therapy, and standardization of malignancy stage for treatment trials and in order to objectively compare therapies among various institutions and over time. ${ }^{61}$ The prognostic factors and the therapeutic approaches to cholangiocarcinoma differ depending upon their location along the biliary tree. Unilateral intrahepatic cholangiocarcinomas are preferentially treated with resection while perihilar cholangiocarcinomas are either treated by resection if they are unilateral or by liver transplantation in selected cases with bilateral hepatic duct involvement. Distal extra-hepatic cholangiocarcinomas frequently require pancreaticoduodenectomy (Whipple procedure), although in rare cases mid common bile duct cancers can be resected without a Whipple procedure. ${ }^{38}$ Identification of the tumor stage and allocation to the optimal treatment approach is important for this aggressive malignancy for which surgery is the only potentially curative treatment. Inaccurate staging can result in inappropriate selection of a treatment option that either deprives patients of a potentially curative procedure or exposes them to unnecessary morbidity and mortality.

\section{Intrahepatic cholangiocarcinoma}

For intrahepatic cholangiocarcinoma, statistically significant prognostic factors confirmed on multivariate analysis include tumor number and differentiation, lymph node metastases, and vascular invasion. ${ }^{62,63}$ Past staging systems (for example, the $6^{\text {th }}$ and earlier editions of the UICC/AJCC staging system) derived their criteria from HCC and therefore also included tumor size as a prognostic factor. However, tumor size as an independent prognostic factor is controversial. Tumor size of $>5 \mathrm{~cm}$ has been shown by several studies to be an independent prognostic factor for survival. ${ }^{63-66}$ However, several other studies have reported that tumor size is not an independent predictor for survival on multivariate analysis. ${ }^{3,7,62,67}$ Regional lymph node metastases are an independent predictor of survival and are, therefore, included in all staging systems. A preoperative serum CA 19-9 level of $>135 \mathrm{U} / \mathrm{ml}$ was reported as a predictor for survival and lymph node metastases on multivariate analysis. ${ }^{67}$ The number of involved lymph nodes has been shown to be important only on univariate analysis but not on multivariate analysis. ${ }^{67}$ A ratio of positive to total harvested lymph nodes of more than 0.2 was suggested as a prognostic factor for overall and recurrence-free survival. ${ }^{63}$

Three staging systems exist: the AJCC/UICC TNM staging system, ${ }^{7}$ the LCSGJ, ${ }^{6}$ and the National Cancer Center of Japan (NCCJ) staging system by Obayashi et al ${ }^{68}$ The major difference between these staging systems lies in the T-staging approach. Most recently, the AJCC/UICC staging system has been revised to its $7^{\text {th }}$ version (Supplementary Box 1 online). The main criticism of the $6^{\text {th }}$ edition was its unspecific application of a staging system originally developed for HCC to intrahepatic cholangiocarcinoma. In particular, 
tumor size as a criterion is problematic, as discussed above. As a result, Nathan et al. demonstrated that the $6^{\text {th }}$ edition of the AJCC staging system was shown to be unable to distinguish prognostically $\mathrm{T} 2$ from $\mathrm{T} 3$ tumors. ${ }^{7}$ In the same study, tumor number and vascular invasion were found to be significant prognostic markers; additive or synergistic prognostic effects of these factors were not observed. Also, lymph node status had prognostic value for survival - patients with more than three involved lymph nodes have a worse prognosis. The validity of the $7^{\text {th }}$ edition AJCC/UICC staging system for intra hepatic cholangiocarcinoma was confirmed in a study that showed significant stage-survival correlation in non-metastatic intrahepatic cholangiocarcinoma in comparison with earlier editions and the NCCJ staging systems. ${ }^{69}$ However, the study was a multicenter, retrospective study with the inherent weaknesses of such an analysis.

The LCSGJ developed another staging system based upon their analysis of prognostic factors in 136 cases of mass-forming intrahepatic cholangiocarcinoma. ${ }^{6}$ Their multivariate analysis showed the following factors to be adverse prognostic factors: tumor size $>2 \mathrm{~cm}$; more than one tumor nodule; and vascular and/or serosal membrane invasion. On the basis of these factors, the LCSGJ staging system was developed (Supplementary Box 2 online). However, several studies have shown that the $\mathrm{T}$ stages in this staging system are not correlated with survival, and omission of serosal invasion improved that correlation; thus, the staging system has been modified. 7,70

In 2001, Obayashi et al. from the NCCJ proposed a new staging system for mass-forming intrahepatic cholangiocarcinoma (Supplementary Box 3 online). ${ }^{68}$ This staging system is based on a multivariate analysis that showed the following factors to be independent prognostic factors: hepatic regional lymph nodes metastases; multiple tumor nodules; symptomatic tumor burden; and vascular invasion. ${ }^{68}$ It is similar to the $7^{\text {th }}$ edition UICC/ AJCC staging system in incorporating tumor number and vascular invasion but not tumor size. However, the study included only 60 patients and the scoring system has not been externally validated.

The $6^{\text {th }}$ edition AJCC/UICC, the LCSGJ and the NCCJ staging systems have been compared. ${ }^{7}$ The $6^{\text {th }}$ edition of the AJCC/UICC system was found to be unable to stratify patients with T2 and T3 tumors to prognostically distinct groups. The LCSGJ system was found to provide no difference in survival between all $\mathrm{T}$ stages, although information on serosal invasion - the T4 stage in the LCSGJ system-was not available for the patients included in this comparative analysis. The same authors proposed a new T-staging system by combining the AJCC/UICC T2 and T3 stage to one T stage (sT2), maintaining the T1stage definition (sT1) and changing the AJCC/UICC T4 stage to sT3; N staging and M staging remained unchanged. The corresponding stages were defined as stage I (sT1NOM0), stage II (sT2NOM0), stage III (sT3N0M0 or N1M0 with any sT) and stage IV (M1 with any sT and N). This system is based upon the observation that there is no significant difference in survival between patients with a single tumor with vascular invasion versus patients with multiple tumors regardless of vascular invasion. Using Cox hazard proportional hazard model analysis, the four systems were compared based upon their T-stage classification and their overall stage grouping. In regard to the T staging, the proposed simplified AJCC/UICC and the Obayashi staging systems performed comparably but were superior to the LCSGJ system. However, in the overall stage grouping they all performed similarly with $c$-indices between 0.65 and 0.68 .

Most recently, Sotiropoulos et al. found gender, AJCC/UICC stage and R0 resection to be the only independent predictors of survival on multivariate analysis and proposed a new scoring system. ${ }^{71}$ On the basis of these criteria, they used a prognostic scoring system allocating one point for advantageous prognostic factors and two for disadvantageous ones 
(female $=1$, male $=2$; stage I-II $+=1$, stage III-IV $=2 ; \mathrm{R} 0=1, \mathrm{R} 1=2$ ). Prognostic stratification into groups with $<5$ and $>5$ points was found to be significant for survival on internal and external validation. A second preoperative clinical scoring system was developed using gender, CA 19-9 levels (100 U/ml cut-off for advantageous versus disadvantageous factor) and macro vascular invasion as prognostic factors. However, the patient sample size was relatively small ( 80 patients) and these scoring systems do not include well-established factors such as vascular invasion and tumor numbers.

In summary, none of the existing staging systems fulfills the criteria of an optimal staging system. In particular, the LCSGJ staging system is inadequate owing to the lack of correlation between the T stages and survival. The $7^{\text {th }}$ edition AJCC/UICC staging system requires histologic evaluation for the $\mathrm{T}$ is (carcinoma in situ) and $\mathrm{T} 4$ stages, and is therefore not reliable for preoperative staging. The proposed staging system by Sotiropoulos et al. largely relies on nonestablished prognostic factors and does not include the majority of the established ones. The NJCC staging system includes all established independent, prognostic factors, and enables preoperative staging but, similar to the other systems, has only a suboptimal correlation with survival. Hence, none of the existing staging systems can be recommended for prognostic information. Similarly, none of these staging systems has demonstrated that they are sufficiently able to stratify patients to treatment arms. Clearly, more work is necessary to optimize the staging systems for intrahepatic cholangiocarcinoma.

\section{Perihilar cholangiocarcinoma}

Widely recognized independent prognostic factors for perihilar cholangiocarcinoma include lymph node metastatic status, tumor differentiation and R0 resection. ${ }^{72-74}$ Several studies also suggest that performance status, comorbidities, and albumin and bilirubin levels are independent prognostic factors. ${ }^{39,75,76}$ Perihilar cholangiocarcinoma is the only type of cholangiocarcinoma that can be treated by liver transplantation with neoadjuvant chemoradiation therapy. Predictors of recurrence after transplantation include old age, CA 19-9 levels $>100 \mathrm{U} / \mathrm{ml}$, mass on cross-sectional imaging, tumor size $>2 \mathrm{~cm}$ in explant, perineural invasion and tumor grade. ${ }^{77}$

One of the first classification systems for perihilar cholangiocarcinoma was the BismuthCorlette classification, which distinguished four types of perihilar cholangiocarcinoma based upon their perihilar extension. This system was originally developed to guide the surgical management of patients with perihilar cholangiocarcinoma. ${ }^{78,79}$ However, this classification system is not a staging system. Its drawbacks include a lack of information regarding vascular encasement, local or distant metastatic spread, lymph node metastases and hepatic lobar atrophy. Hence, this system does not yield prognostic value and does not correlate with outcomes. ${ }^{76,80}$ In addition, it is not applicable for patients with biliary normal variants, such as trifurcations.

The Memorial Sloan-Kettering Cancer Center (MSKCC) staging system by Blumgart and colleagues classifies tumors according to their location and extent of bile duct involvement (similar to the Bismuth-Corlette classification), portal venous invasion, and hepatic lobar atrophy (Supplementary Table 1 online) ${ }^{81}$ It was derived using data from 219 patients with perihilar cholangiocarcinoma treated by resection and serves as a preoperative staging system to determine resectability. Per postoperative analysis, the $\mathrm{T}$ stages were significantly correlated to R0, N2 and M1 status and survival. However, 160 patients in this study were deemed potential candidates for resection and underwent exploration; 80 of these 160 patients were found to have unresectable disease during surgery. Zervos et al. re-evaluated an earlier Blumgart staging system and did not find a correlation between the $\mathrm{T}$ stages and resectability and survival. ${ }^{80}$ 
In the updated $7^{\text {th }}$ edition AJCC/UICC staging system, perihilar cholangiocarcinoma is separated from distal extrahepatic cholangiocarcinoma and staged as its own entity (Supplementary Box 4 online). This is an important correction as intrahepatic, perihilar and distal extrahepatic cholangiocarcinomas differ in their presentation, natural history, management and molecular signature. ${ }^{38}$ Occasionally, there may be an overlap between perihilar and intrahepatic cholangiocarcinoma involving the major hepatic ducts. Further division of perihilar cholangiocarcinoma into intrahepatic cholangiocarcinoma involving the hilum versus hilar cholangiocarcinoma has been evaluated but no stage specific survival benefit between these two anatomical tumor types has been demonstrated; thus a further subdivision into these two types is not recommended. ${ }^{82,83}$ Moreover, we find this distinction - that is, intrahepatic cholangiocarcinoma involving the hilum versus perihilar cholangiocarcinoma - to be artificial and without biologic or clinical meaning.

A new staging system for perihilar cholangiocarcinoma has been proposed (Supplementary Table 2 online). ${ }^{84}$ This staging system is based upon published prognostic factors, resectability criteria and personal experience. It includes size and number of the primary tumor, local and distant metastatic disease, vascular encasement and lobar atrophy; in addition it uses presurgical clinical parameters such as cholestasis and its response to stenting as well as the patient's performance status. At this point, the staging system still requires validation but, in our opinion, will be able to provide presurgical prognostic information and guide the therapeutic approach.

Most recently, an international cholangiocarcinoma working group consisting of leaders in the field suggested a new staging system from a surgical perspective. ${ }^{2}$ The proposed staging classification is derived from components of the Bismuth-Corlette classification, and the TNM and MSKCC staging systems but also includes new factors. It uses eight characteristics for staging: tumor extent; tumor size, tumor growth type; vascular involvement; hepatic lobar atrophy; under lying hepatic disease; lymph node metastases; and distant metastases (Supplementary Table 3 online). Hence, this staging system is applicable in the preoperative setting and includes established prognostic factors. However, it is rather complicated and also includes some prognostic factors that have not been established ${ }^{81}$ For example, several studies have shown that tumor size is not a statistically independent prognostic factor. ${ }^{81,85}$ Lobar atrophy has been shown to be of prognostic value in univariate but not multivariate analysis. ${ }^{81}$ Hence, the volumetric criterion is more of a factor for resection rather than of prognostic value. The inclusion of the macroscopic tumor growth type is a new approach and has not been included in other staging systems; however, it has been shown to be a predictive factor for survival. ${ }^{9,86}$ Additional points of criticism have included the criteria for the vascular involvement, such as the requirement of 180 degrees involvement and the derivation of the vascular from the biliary involvement. ${ }^{87}$ Hence, the validity of this system will require confirmation in large prospective studies and the authors have opened a registry in order to validate their system.

In summary, there is currently no optimal staging system for perihilar cholangiocarcinoma. The existing ones can be helpful for the surgical decision process but either lack significant clinical information or have been shown to be unreliable in their predictive ability to select patients who should undergo resection. Other staging systems are prognostically unreliable. However, new systems are under development and their validation in larger study populations is awaited.

\section{Distal extrahepatic cholangiocarcinoma}

The $7^{\text {th }}$ edition AJCC/UICC TNM staging classification is the first classification system that has assigned a separate staging system for distal extrahepatic cholangiocarcinoma (Supplementary Box 5 online). Distal extrahepatic cholangiocarcinoma is defined as 
involving the common bile duct from the cystic duct to the ampulla of Vater. This is an important step as differences between these two extrahepatic forms of cholangiocarcinoma have been shown. For example, depth of invasion and pancreatic invasion are significantly more common in distal extra-hepatic cholangiocarcinoma. ${ }^{88}$ For distal extrahepatic cholangiocarcinoma, tumor depth invasion, lymph node metastases, perineural, microscopic vascular invasion as well as invasion in the pancreas, and R0 resection were found to be significant predictors of survival. ${ }^{72,89-91}$ Age, gender, tumor histology and histologic grading were not found to be statistically prognostic factors. Lymph node metastatic spread differs between the three types of cholangiocarcinoma and is most commonly observed in distal extrahepatic cholangio carcinoma. ${ }^{92}$ Several studies have suggested the number of lymph nodes to be an independent prognostic factor; more than two metastatic lymph nodes is predictive of worse outcomes. ${ }^{93,94}$

The T stage of the AJCC classification distinguishes T1 and T2 tumors based upon microscopic tumor growth confined to the bile duct versus beyond it - a definition frequently criticized for its vagueness. Accordingly, the AJCC/UICC T stages were not found to be predictors of outcomes after resection of these cancers, which indicates that the definition needs to be more precise. One study grouped tumors into three groups according to their invasion depth: $<5 \mathrm{~mm}, 5-12 \mathrm{~mm}$ and $>12 \mathrm{~mm}$. They found that tumor depth was the strongest predictor for outcome on multivariate analysis. ${ }^{89}$ Also, T3 and T4 stages have been redefined, with T4 describing invasion in the celiac axis and superior mesenteric artery, and T3 describing invasion of adjacent organs but without invasion of arterial structures. The prior AJCC/UICC distinguished T3 and T4 stages based upon invasion of different organs; however, it was shown that with this definition of T3 and T4 was not able to distinguish prognosis. ${ }^{95}$ Hence, the separation by the $7^{\text {th }}$ edition into distal and perihilar extrahepatic cholangiocarcinoma is a major improvement compared with prior staging systems. Redefinition of the $\mathrm{T}$ stages is also a step in the right direction.

In summary, the $7^{\text {th }}$ edition AJCC/UICC staging system is currently the only existing staging system for distal extrahepatic cholangiocarcinoma. Its purpose is predominantly prognostic and it will require additional validation. A staging system providing treatment guidance has not been developed and is needed.

\section{Conclusions}

Cholangiocarcinoma is a devastating malignancy with an increasing incidence. It is difficult to diagnose and frequently presents at a late stage. The diagnostic criteria described above are derived from established data, and the proposed diagnostic approach should provide sufficient guidance for diagnosis and management. In addition, the fact that some intrahepatic cholangiocarcinomas that occur in chronic liver disease can mimic $\mathrm{HCC}$ on imaging needs addressing. There is growing acceptance that tumors should be treated differently according to their location. One of the weaknesses of existing staging systems is their reliance on histopathologic criteria, which thereby limits their preoperative use. However, important progress has been made in the modification of staging systems whose validity will hopefully be confirmed in large prospective studies.

\section{Supplementary Material}

Refer to Web version on PubMed Central for supplementary material.

\section{Acknowledgments}

This work was supported in part by grant DK59427 from the NIH (G. J. Gores), the Mayo Clinic Clinical Investigator Program (B. Blechacz) and the Mayo Foundation. 


\section{References}

1. Everhart JE, Ruhl CE. Burden of digestive diseases in the United States Part III: liver, biliary tract, and pancreas. Gastroenterology. 2009; 136:1134-1144. [PubMed: 19245868]

2. DeOliveira ML, et al. New staging system and a registry for perihilar cholangiocarcinoma. Hepatology. 2011; 53:1363-1371. [PubMed: 21480336]

3. DeOliveira ML, et al. Cholangiocarcinoma: thirty-one-year experience with 564 patients at a single institution. Ann Surg. 2007; 245:755-762. [PubMed: 17457168]

4. Welzel TM, McGlynn KA, Hsing AW, O'Brien TR, Pfeiffer RM. Impact of classification of hilar cholangiocarcinomas (Klatskin tumors) on the incidence of intra- and extrahepatic cholangiocarcinoma in the United States. J Natl Cancer Inst. 2006; 98:873-875. [PubMed: 16788161]

5. Burak K, et al. Incidence and risk factors for cholangiocarcinoma in primary sclerosing cholangitis. Am J Gastroenterol. 2004; 99:523-526. [PubMed: 15056096]

6. Yamasaki S. Intrahepatic cholangiocarcinoma: macroscopic type and stage classification. J Hepatobiliary Pancreat Surg. 2003; 10:288-291. [PubMed: 14598147]

7. Nathan H, et al. A proposed staging system for intrahepatic cholangiocarcinoma. Ann Surg Oncol. 2009; 16:14-22. [PubMed: 18987916]

8. Sasaki A, et al. Intrahepatic peripheral cholangiocarcinoma: mode of spread and choice of surgical treatment. Br J Surg. 1998; 85:1206-1209. [PubMed: 9752860]

9. Shimada K, et al. Surgical outcomes of the mass-forming plus periductal infiltrating types of intrahepatic cholangiocarcinoma: a comparative study with the typical mass-forming type of intrahepatic cholangiocarcinoma. World J Surg. 2007; 31:2016-2022. [PubMed: 17687597]

10. Yamamoto $\mathrm{Y}$, et al. Clinicopathological characteristics of intrahepatic cholangiocellular carcinoma presenting intrahepatic bile duct growth. J Surg Oncol. 2009; 99:161-165. [PubMed: 19101954]

11. Farges $\mathrm{O}$, et al. AJCC 7th edition of TNM staging accurately discriminates outcomes of patients with resectable intrahepatic cholangiocarcinoma: by the AFC-IHCC-2009 study group. Cancer. 2010; 117:2170-2177. [PubMed: 21523730]

12. El Rassi ZE, et al. Peripheral cholangiocarcinoma: presentation, diagnosis, pathology and management. Eur J Surg Oncol. 1999; 25:375-380. [PubMed: 10419707]

13. Torzilli G, et al. Accurate preoperative evaluation of liver mass lesions without fine-needle biopsy. Hepatology. 1999; 30:889-893. [PubMed: 10498639]

14. Blechacz B, Gores GJ. Positron emission tomography scan for a hepatic mass. Hepatology. 2010; 52:2186-2191. [PubMed: 20967825]

15. Vilgrain V, et al. Intrahepatic cholangiocarcinoma: MRI and pathologic correlation in 14 patients. $\mathrm{J}$ Comput Assist Tomogr. 1997; 21:59-65. [PubMed: 9022771]

16. Braga HJ, Imam K, Bluemke DA. MR imaging of intrahepatic cholangiocarcinoma: use of ferumoxides for lesion localization and extension. AJR Am J Roentgenol. 2001; 177:111-114. [PubMed: 11418408]

17. Raman SS, et al. Hepatic MR imaging using ferumoxides: prospective evaluation with surgical and intraoperative sonographic confirmation in 25 cases. AJR Am J Roentgenol. 2001; 177:807-812. [PubMed: 11566677]

18. Rimola J, et al. Cholangiocarcinoma in cirrhosis: absence of contrast washout in delayed phases by magnetic resonance imaging avoids misdiagnosis of hepatocellular carcinoma. Hepatology. 2009; 50:791-798. [PubMed: 19610049]

19. Vilgrain V. Staging cholangiocarcinoma by imaging studies. HPB (Oxford). 2008; 10:106-109. [PubMed: 18773065]

20. Corvera CU, et al. $18 \mathrm{~F}$-fluorodeoxyglucose positron emission tomography influences management decisions in patients with biliary cancer. J Am Coll Surg. 2008; 206:57-65. [PubMed: 18155569]

21. Tao LY, Cai L, He XD, Liu W, Qu Q. Comparison of serum tumor markers for intrahepatic cholangiocarcinoma and hepatocellular carcinoma. Am Surg. 2010; 76:1210-1213. [PubMed: 21140686]

22. Uenishi T, et al. Cytokeratin-19 fragments in serum (CYFRA 21-1) as a marker in primary liver cancer. Br J Cancer. 2003; 88:1894-1899. [PubMed: 12799633] 
23. Uenishi T, et al. Serum cytokeratin 19 fragment (CYFRA21-1) as a prognostic factor in intrahepatic cholangiocarcinoma. Ann Surg Oncol. 2008; 15:583-589. [PubMed: 17955299]

24. Nakanuma, Y., et al. Intrahepatic cholangiocarcinoma. International Agency for Research on Cancer [IARC]; Lyon: 2010. p. 217-224.

25. Desmet, V.; Roskams, T.; De Vos, R. Normal Anatomy. In: LaRusso, NF., editor. Gastroenterology and Hepatology The Comprehensive Visual Reference. Current Medicine; Philadelphia: 1997. 1.14

26. Nakanuma Y, Hoso M, Sanzen T, Sasaki M. Microstructure and development of the normal and pathologic biliary tract in humans, including blood supply. Microsc Res Tech. 1997; 38:552-570. [PubMed: 9330346]

27. Komuta M, et al. Clinicopathological study on cholangiolocellular carcinoma suggesting hepatic progenitor cell origin. Hepatology. 2008; 47:1544-1556. [PubMed: 18393293]

28. Asayama Y, et al. Imaging of cholangiolocellular carcinoma of the liver. Eur J Radiol. 2010; 75:e120-e125. [PubMed: 19781883]

29. Motosugi U, et al. Cholangiolocellular carcinoma of the liver: imaging findings. J Comput Assist Tomogr. 2009; 33:682-688. [PubMed: 19820492]

30. Rullier A, et al. Cytokeratin 7 and 20 expression in cholangiocarcinomas varies along the biliary tract but still differs from that in colorectal carcinoma metastasis. Am J Surg Pathol. 2000; 24:870-876. [PubMed: 10843291]

31. Maeda T, et al. The expression of cytokeratins 7, 19, and 20 in primary and metastatic carcinomas of the liver. Mod Pathol. 1996; 9:901-909. [PubMed: 8878022]

32. Vilana R, et al. Intrahepatic peripheral cholangiocarcinoma in cirrhosis patients may display a vascular pattern similar to hepatocellular carcinoma on contrast-enhanced ultrasound. Hepatology. 2010; 51:2020-2029. [PubMed: 20512990]

33. Sapisochin G, Fidelman N, Roberts JP, Yao FY. Mixed hepatocellular-cholangiocarcinoma (HCC$\mathrm{CC})$ and intra-hepatic cholangiocarcinoma (I-CC) in patients transplanted for hepatocellular carcinoma. Liver Transpl. 10.1002/lt.22307

34. Yamashita Y, Takahashi M, Kanazawa S, Charnsangavej C, Wallace S. Hilar cholangiocarcinoma. An evaluation of subtypes with CT and angiography. Acta Radiol. 1992; 33:351-355. [PubMed: 1321653]

35. Alden ME, et al. Cholangiocarcinoma: clinical significance of tumor location along the extrahepatic bile duct. Radiology. 1995; 197:511-516. [PubMed: 7480704]

36. Johnson SR, Kelly BS, Pennington LJ, Hanto DW. A single center experience with extrahepatic cholangiocarcinomas. Surgery. 2001; 130:584-590. [PubMed: 11602888]

37. Heron DE, et al. Cholangiocarcinoma: the impact of tumor location and treatment strategy on outcome. Am J Clin Oncol. 2003; 26:422-428. [PubMed: 12902899]

38. Blechacz B, Gores GJ. Cholangiocarcinoma: advances in pathogenesis, diagnosis, and treatment. Hepatology. 2008; 48:308-321. [PubMed: 18536057]

39. Nagorney DM, Donohue JH, Farnell MB, Schleck CD, Ilstrup DM. Outcomes after curative resections of cholangiocarcinoma. Arch Surg. 1993; 128:871-877. [PubMed: 8393652]

40. Hann LE, et al. Hepatic lobar atrophy: association with ipsilateral portal vein obstruction. AJR Am J Roentgenol. 1996; 167:1017-1021. [PubMed: 8819404]

41. Nakeeb A, et al. Cholangiocarcinoma. A spectrum of intrahepatic, perihilar, and distal tumors. Ann Surg. 1996; 224:463-473. [PubMed: 8857851]

42. Ghazale A, et al. Immunoglobulin G4-associated cholangitis: clinical profile and response to therapy. Gastroenterology. 2008; 134:706-715. [PubMed: 18222442]

43. Akdogan M, et al. Extraordinarily elevated CA19-9 in benign conditions: a case report and review of the literature. Tumori. 2001; 87:337-339. [PubMed: 11765186]

44. Levy C, et al. The value of serum CA 19-9 in predicting cholangiocarcinomas in patients with primary sclerosing cholangitis. Dig Dis Sci. 2005; 50:1734-1740. [PubMed: 16133981]

45. Patel AH, Harnois DM, Klee GG, LaRusso NF, Gores GJ. The utility of CA 19-9 in the diagnoses of cholangiocarcinoma in patients without primary sclerosing cholangitis. Am J Gastroenterol. 2000; 95:204-207. [PubMed: 10638584] 
46. Vestergaard EM, et al. Reference values and biological variation for tumor marker CA 19-9 in serum for different Lewis and secretor genotypes and evaluation of secretor and Lewis genotyping in a Caucasian population. Clin Chem. 1999; 45:54-61. [PubMed: 9895338]

47. Feydy A, et al. Helical CT assessment in hilar cholangiocarcinoma: correlation with surgical and pathologic findings. AJR Am J Roentgenol. 1999; 172:73-77. [PubMed: 9888743]

48. Lee HY, et al. Preoperative assessment of resectability of hepatic hilar cholangiocarcinoma: combined CT and cholangiography with revised criteria. Radiology. 2006; 239:113-121. [PubMed: 16467211]

49. Aloia TA, et al. High-resolution computed tomography accurately predicts resectability in hilar cholangiocarcinoma. Am J Surg. 2007; 193:702-706. [PubMed: 17512280]

50. Tillich M, Mischinger HJ, Preisegger KH, Rabl H, Szolar DH. Multiphasic helical CT in diagnosis and staging of hilar cholangiocarcinoma. AJR Am J Roentgenol. 1998; 171:651-658. [PubMed: 9725291]

51. Kato T, et al. Clinical role of (18)F-FDG PET for initial staging of patients with extrahepatic bile duct cancer. Eur J Nucl Med Mol Imaging. 2002; 29:1047-1054. [PubMed: 12173019]

52. Lopera JE, Soto JA, Munera F. Malignant hilar and perihilar biliary obstruction: use of MR cholangiography to define the extent of biliary ductal involvement and plan percutaneous interventions. Radiology. 2001; 220:90-96. [PubMed: 11425978]

53. Manfredi R, Barbaro B, Masselli G, Vecchioli A, Marano P. Magnetic resonance imaging of cholangiocarcinoma. Semin Liver Dis. 2004; 24:155-164. [PubMed: 15192788]

54. Masselli G, Manfredi R, Vecchioli A, Gualdi G. MR imaging and MR cholangiopancreatography in the preoperative evaluation of hilar cholangiocarcinoma: correlation with surgical and pathologic findings. Eur Radiol. 2008; 18:2213-2221. [PubMed: 18463877]

55. Vogl TJ, et al. Staging of Klatskin tumours (hilar cholangiocarcinomas): comparison of MR cholangiography, MR imaging, and endoscopic retrograde cholangiography. Eur Radiol. 2006; 16:2317-2325. [PubMed: 16622690]

56. Kluge R, et al. Positron emission tomography with [(18)F]fluoro-2-deoxy-D-glucose for diagnosis and staging of bile duct cancer. Hepatology. 2001; 33:1029-1035. [PubMed: 11343227]

57. Hattori M, et al. Prospective study of biliary cytology in suspected perihilar cholangiocarcinoma. Br J Surg. 2011; 98:704-709. [PubMed: 21290384]

58. Halling KC, Kipp BR. Fluorescence in situ hybridization in diagnostic cytology. Hum Pathol. 2007; 38:1137-1144. [PubMed: 17640552]

59. DeHaan RD, et al. An assessment of chromosomal alterations detected by fluorescence in situ hybridization and p16 expression in sporadic and primary sclerosing cholangitis-associated cholangiocarcinomas. Hum Pathol. 2007; 38:491-499. [PubMed: 17239935]

60. Lee M, Banerjee S, Posner MC, Cartwright CA. Distal extrahepatic cholangiocarcinoma presenting as cholangitis. Dig Dis Sci. 2010; 55:1852-1855. [PubMed: 20499173]

61. Sobin LH. TNM: principles, history, and relation to other prognostic factors. Cancer. 2001; 91:1589-1592. [PubMed: 11309755]

62. Choi SB, et al. The prognosis and survival outcome of intrahepatic cholangiocarcinoma following surgical resection: association of lymph node metastasis and lymph node dissection with survival. Ann Surg Oncol. 2009; 16:3048-3056. [PubMed: 19626372]

63. Tamandl D, et al. Lymph node ratio after curative surgery for intrahepatic cholangiocarcinoma. $\mathrm{Br}$ J Surg. 2009; 96:919-925. [PubMed: 19591163]

64. Endo I, et al. Intrahepatic cholangiocarcinoma: rising frequency, improved survival, and determinants of outcome after resection. Ann Surg. 2008; 248:84-96. [PubMed: 18580211]

65. Shen WF, et al. Clinicopathological and prognostic analysis of 429 patients with intrahepatic cholangiocarcinoma. World J Gastroenterol. 2009; 15:5976-5982. [PubMed: 20014463]

66. Weber SM, et al. Intrahepatic cholangiocarcinoma: resectability, recurrence pattern, and outcomes. J Am Coll Surg. 2001; 193:384-391. [PubMed: 11584966]

67. Uchiyama K, et al. Impact of nodal involvement on surgical outcomes of intrahepatic cholangiocarcinoma: a multicenter analysis by the Study Group for Hepatic Surgery of the Japanese Society of Hepato-Biliary-Pancreatic Surgery. J Hepatobiliary Pancreat Sci. 2011; 18:443-452. [PubMed: 21132443] 
68. Okabayashi T, et al. A new staging system for mass-forming intrahepatic cholangiocarcinoma: analysis of preoperative and postoperative variables. Cancer. 2001; 92:2374-2383. [PubMed: 11745293]

69. Farges O, et al. AJCC 7th edition of TNM staging accurately discriminates outcomes of patients with resectable intrahepatic cholangiocarcinoma: by the AFC-IHCC-2009 study group. Cancer. 2011; 117:2170-2177. [PubMed: 21523730]

70. Uenishi T, et al. Serosal invasion in TNM staging of mass-forming intrahepatic cholangiocarcinoma. J Hepatobiliary Pancreat Surg. 2005; 12:479-483. [PubMed: 16365823]

71. Sotiropoulos GC, et al. Multicentric evaluation of a clinical and prognostic scoring system predictive of survival after resection of intrahepatic cholangiocarcinomas. Liver Int. 2010; 30:9961002. [PubMed: 20141593]

72. Ito K, et al. Adequate lymph node assessment for extrahepatic bile duct adenocarcinoma. Ann Surg. 2010; 251:675-681. [PubMed: 20224368]

73. Klempnauer J, et al. Resectional surgery of hilar cholangiocarcinoma: a multivariate analysis of prognostic factors. J Clin Oncol. 1997; 15:947-954. [PubMed: 9060532]

74. Ramacciato G, et al. Univariate and multivariate analysis of prognostic factors in the surgical treatment of hilar cholangiocarcinoma. Am Surg. 2010; 76:1260-1268. [PubMed: 21140696]

75. Fernandez-Ruiz M, Guerra-Vales JM, Colina-Ruizdelgado F. Comorbidity negatively influences prognosis in patients with extrahepatic cholangiocarcinoma. World J Gastroenterol. 2009; 15:5279-5286. [PubMed: 19908335]

76. Park J, et al. Natural history and prognostic factors of advanced cholangiocarcinoma without surgery, chemotherapy, or radiotherapy: a large-scale observational study. Gut Liver. 2009; 3:298305. [PubMed: 20431764]

77. Heimbach JK, et al. Predictors of disease recurrence following neoadjuvant chemoradiotherapy and liver transplantation for unresectable perihilar cholangiocarcinoma. Transplantation. 2006; 82:1703-1707. [PubMed: 17198263]

78. Bismuth H, Corlette MB. Intrahepatic cholangioenteric anastomosis in carcinoma of the hilus of the liver. Surg Gynecol Obstet. 1975; 140:170-178. [PubMed: 1079096]

79. Bismuth H, Nakache R, Diamond T. Management strategies in resection for hilar cholangiocarcinoma. Ann Surg. 1992; 215:31-38. [PubMed: 1309988]

80. Zervos EE, et al. Stage does not predict survival after resection of hilar cholangiocarcinomas promoting an aggressive operative approach. Am J Surg. 2005; 190:810-815. [PubMed: 16226963]

81. Jarnagin WR, et al. Staging, resectability, and outcome in 225 patients with hilar cholangiocarcinoma. Ann Surg. 2001; 234:507-517. [PubMed: 11573044]

82. Ebata T, et al. The concept of perihilar cholangiocarcinoma is valid. Br J Surg. 2009; 96:926-934. [PubMed: 19591162]

83. Sano T, et al. Prognosis of perihilar cholangiocarcinoma: hilar bile duct cancer versus intrahepatic cholangiocarcinoma involving the hepatic hilus. Ann Surg Oncol. 2008; 15:590-599. [PubMed: 18057991]

84. Blechacz BR, Sanchez W, Gores GJ. A conceptual proposal for staging ductal cholangiocarcinoma. Curr Opin Gastroenterol. 2009; 25:238-239. [PubMed: 19387257]

85. Ito F, et al. Resection of hilar cholangiocarcinoma: concomitant liver resection decreases hepatic recurrence. Ann Surg. 2008; 248:273-279. [PubMed: 18650638]

86. Guglielmi A, et al. Intrahepatic cholangiocarcinoma: prognostic factors after surgical resection. World J Surg. 2009; 33:1247-1254. [PubMed: 19294467]

87. Nagino M. Perihilar cholangiocarcinoma: a much needed but imperfect new staging system. Nat Rev Gastroenterol Hepatol. 2010; 8:252-253. [PubMed: 21487382]

88. Sakamoto Y, et al. Prognostic factors of surgical resection in middle and distal bile duct cancer: an analysis of 55 patients concerning the significance of ductal and radial margins. Surgery. 2005; 137:396-402. [PubMed: 15800484]

89. Hong SM, et al. Depth of tumor invasion better predicts prognosis than the current American Joint Committee on Cancer T classification for distal bile duct carcinoma. Surgery. 2009; 146:250-257. [PubMed: 19628081] 
90. Murakami Y, et al. Prognostic significance of lymph node metastasis and surgical margin status for distal cholangiocarcinoma. J Surg Oncol. 2007; 95:207-212. [PubMed: 17278119]

91. Woo SM, et al. Recurrence and prognostic factors of ampullary carcinoma after radical resection: comparison with distal extrahepatic cholangiocarcinoma. Ann Surg Oncol. 2007; 14:3195-3201. [PubMed: 17710498]

92. Yoshida T, et al. Lymphatic spread differs according to tumor location in extrahepatic bile duct cancer. Hepatogastroenterology. 2003; 50:17-20. [PubMed: 12629981]

93. Murakami Y, et al. Pancreatoduodenectomy for distal cholangiocarcinoma: prognostic impact of lymph node metastasis. World J Surg. 2007; 31:337-342. [PubMed: 17006609]

94. Yoshida T, et al. Prognostic factors after pancreatoduodenectomy with extended lymphadenectomy for distal bile duct cancer. Arch Surg. 2002; 137:69-73. [PubMed: 11772220]

95. Ebata T, et al. Pancreatic and duodenal invasion in distal bile duct cancer: paradox in the tumor classification of the American Joint Committee on Cancer. World J Surg. 2007; 31:2008-2015. [PubMed: 17661132] 


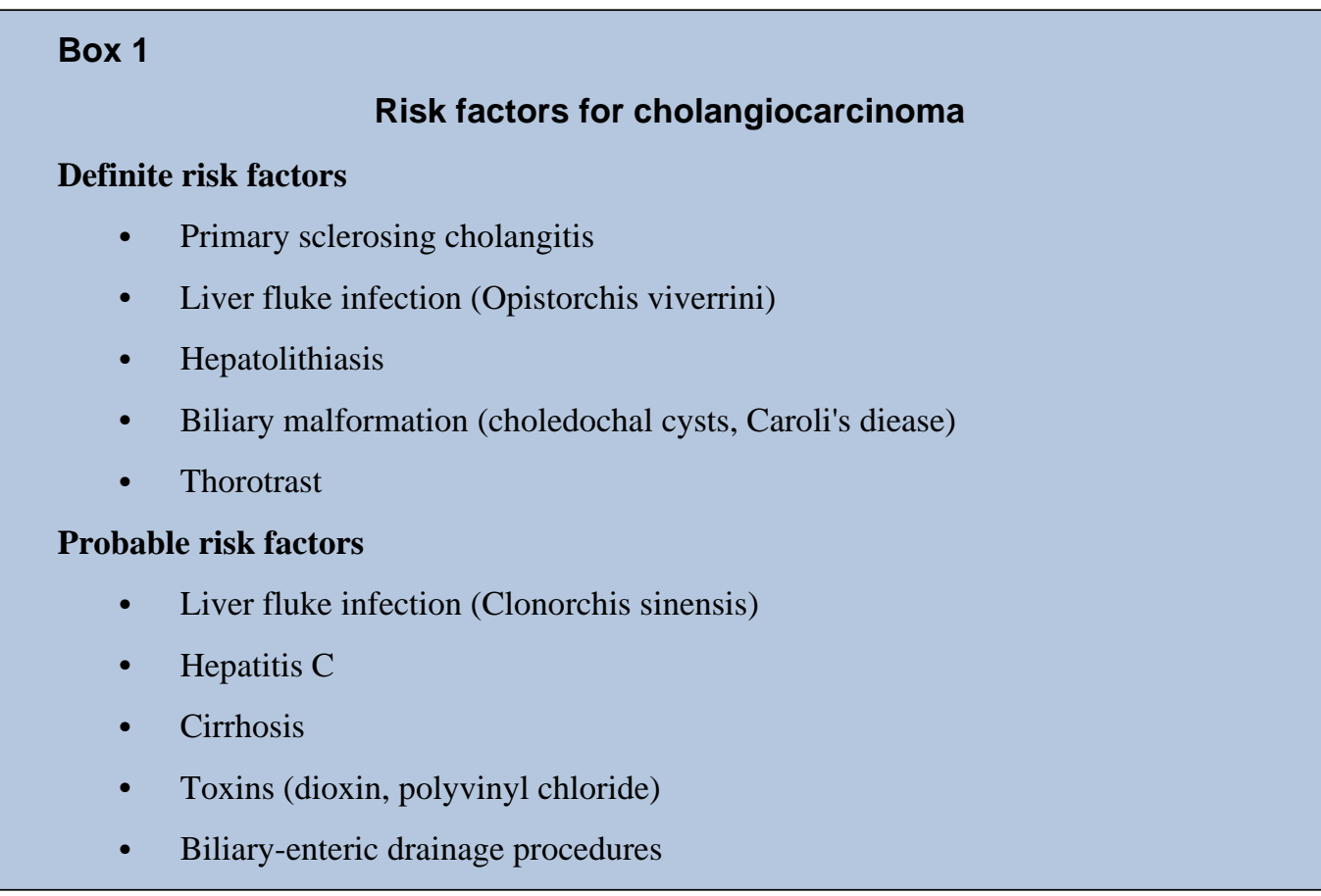




\section{Box 2 \\ Histologic type of cholangiocarcinomas}

Adenocarcinoma

Clear cell adenocarcinoma

Mucinous carcinoma

Signet ring cell carcinoma

Squamous cell carcinoma

Adenosquamous carcinoma

Small cell carcinoma

- Undifferentiated carcinoma

- Spindle and giant cell types

Small cell types

Papillomatosis

Papillary carcinoma, noninvasive

Papillary carcinoma, invase

Carcinoma 


\section{Key points}

- Cholangiocarcinoma is classified according to its anatomic location into three subtypes: intrahepatic, perihilar and distal extrahepatic cholangiocarcinoma

- The diagnosis of intrahepatic cholangiocarcinoma requires histopathology and is a diagnosis of exclusion; a pathologic staging system for this cholangiocarcinoma subtype has been proposed

- The diagnosis of perihilar cholangiocarcinoma is often made clinically, and is aided by cytologic fluorescent in situ hybridization studies; staging systems for this subtype of cholangiocarcinoma are still evolving

- A diagnosis of distal extrahepatic cholangiocarcinoma can usually be confirmed by cytology; stage is highly dependent upon depth of invasion of surrounding structures 


\section{Review criteria}

We searched PubMed using the following terms: "bile duct cancer", "biliary tract cancer", and "cholangiocarcinoma". Search dates included January 11990 to March 1 2011. Only full-text papers in English were selected for the Review. The reference lists of the retrieved manuscripts were searched for additional pertinent references. 


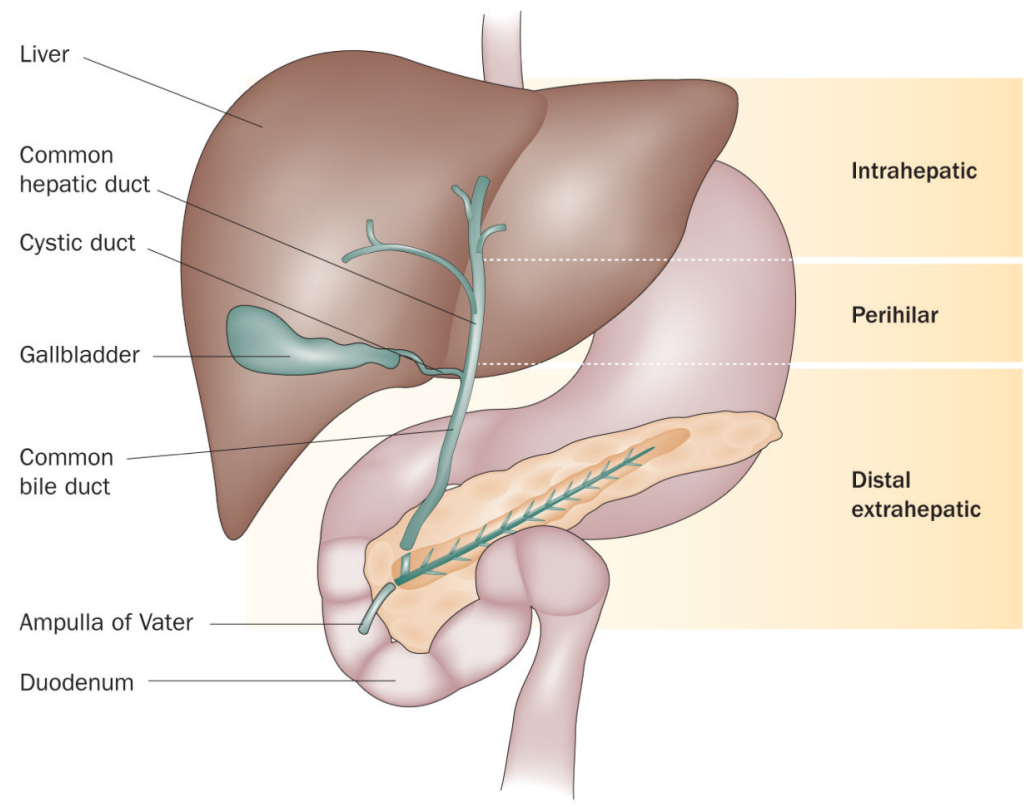

Figure 1.

Cholangiocarcinoma subtypes. Cholangiocarcinoma is classified as either intrahepatic or extrahepatic, with the second-order bile ducts acting as the separation point. Classically, extrahepatic cholangiocarcinoma has been divided in perihilar and distal extrahepatic cholangiocarcinoma at the level of the cystic duct. 

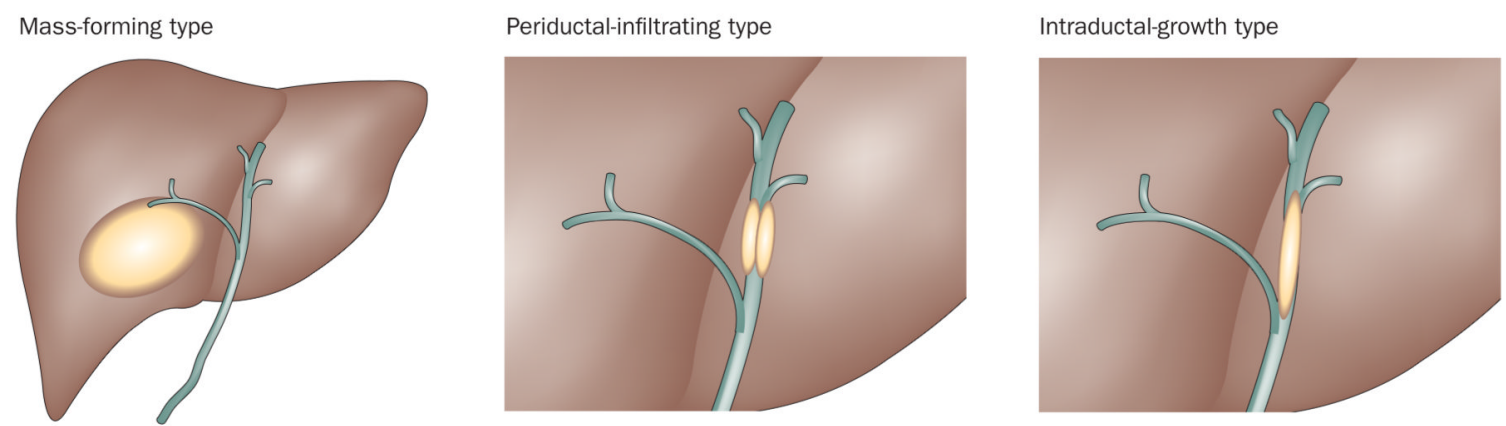

Figure 2.

Intrahepatic cholangiocarcinoma growth types. The Liver Cancer Study Group of Japan (LCSGJ) used retrospective data from 245 patients to distinguish three macroscopic growth types for intrahepatic cholangiocarcinoma: mass-forming type; periductal-infiltrating type; and intraductal-growth type. 


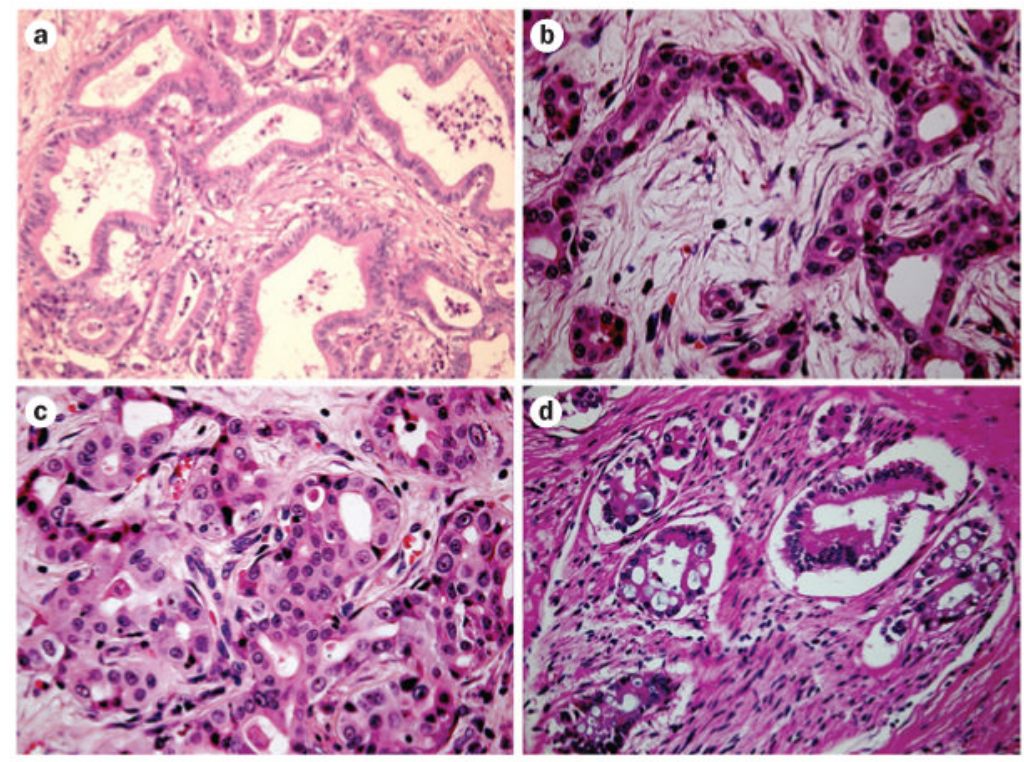

Figure 3.

Histpathological features of mucin-producing cholangiocarcinoma and cholangiolocellular carcinoma with mixed features. a | Well differentiated intrahepatic cholangiocarcinoma. Tubular structure with abundant fibrous stroma. $\mathbf{b} \mid$ Cholangiolocellular carcinoma. Ductular-reaction-like structure with fine edematous fibrous stroma. $\mathbf{c} \mid$ Hepatocellularcarcinoma-like area in cholangiolocellular carcinoma. $\mathbf{d} \mid$ Mucin-producing perihilar cholangiocarcinoma showing perineural invasion. 


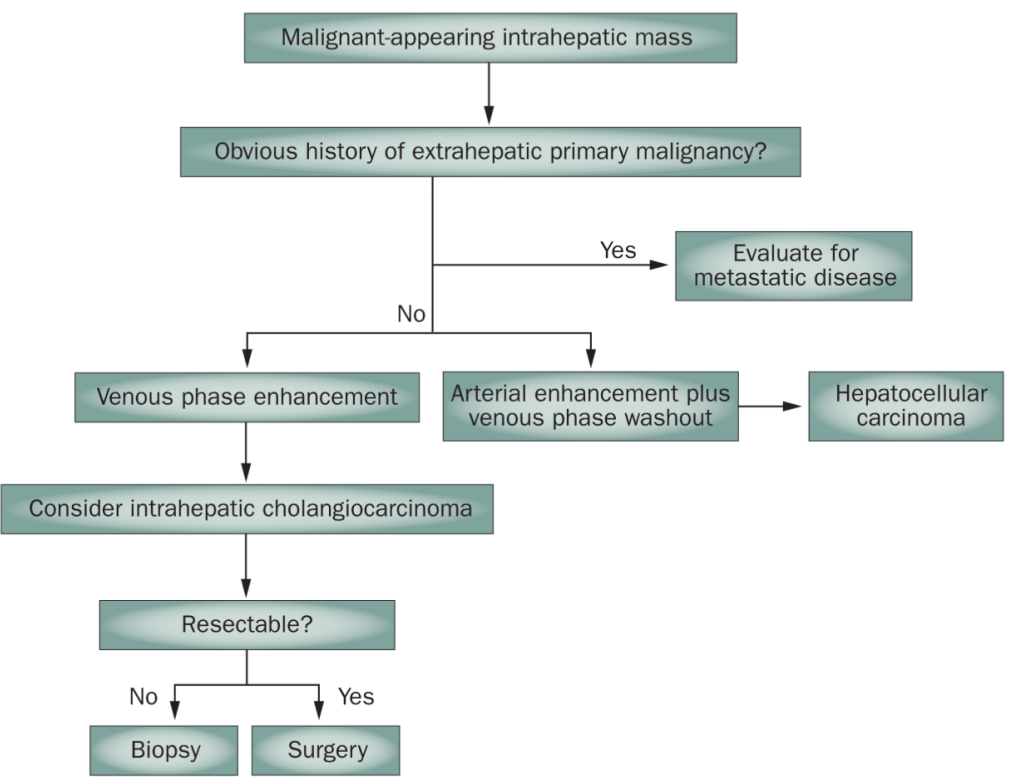

Figure 4.

Algorithm for the diagnosis and management of intrahepatic cholangiocarcinoma. 


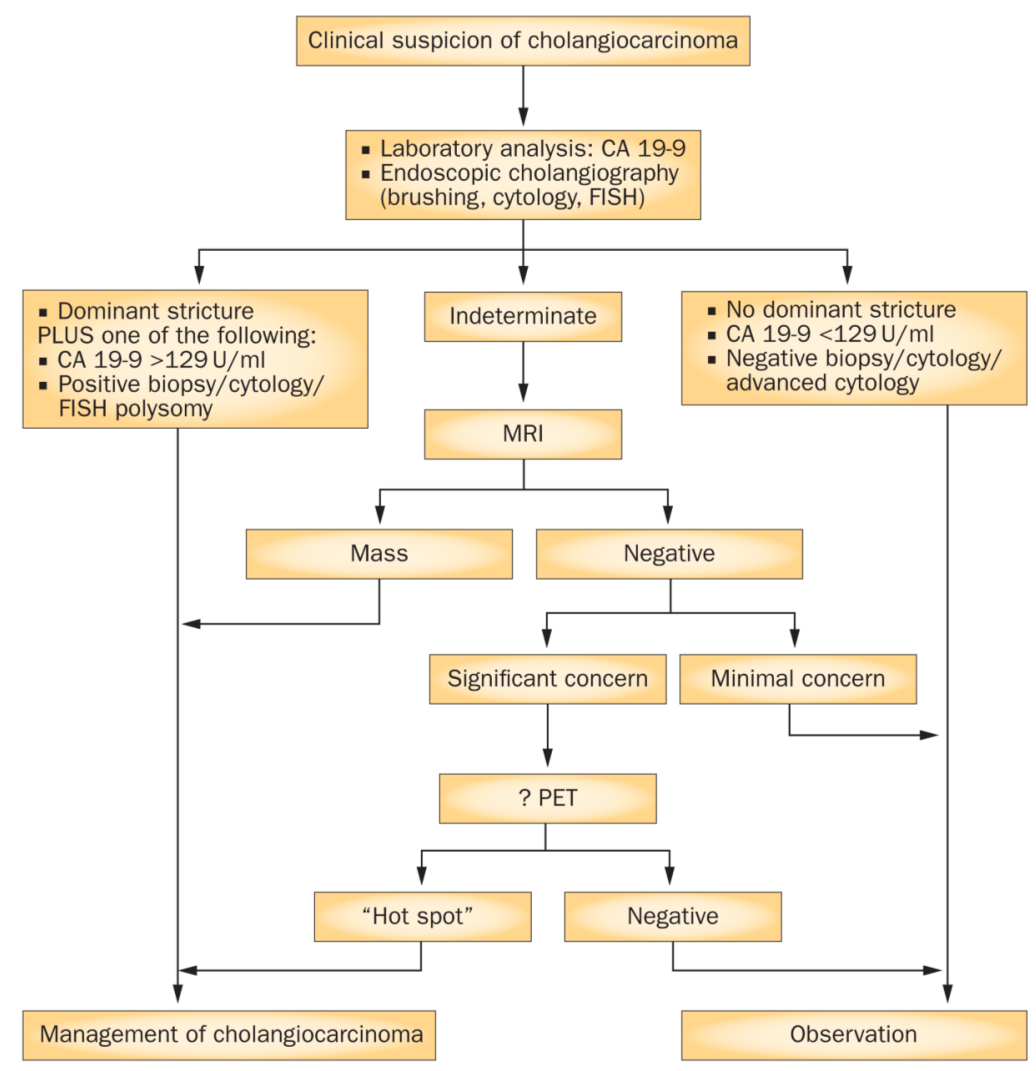

Figure 5.

Diagnostic criteria for perihilar and distal extrahepatic cholangiocarcinoma. 